\title{
SANDSTONE WATER SENSITIVITY: EXISTENCE OF A CRITICAL RATE OF SALINITY DECREASE FOR PARTICLE CAPTURE
}

\author{
K. C. KHILAR $\dagger$, H. S. FOGLER* and J. S. AHLUWALIA \\ Department of Chemical Engineering, The University of Michigan, Ann Arbor, MI 48109, U.S.A.
}

(Received 14 December 1981; accepted 1 September 1982)

\begin{abstract}
Alstract-A critical rate of salinity decrease (CRSD) was found to exist in the water sensitivity of Berea sandstone. The CRSD was determined by conducting core flood experiments in which the salinity was decreased exponentially by placing mixers of various volumes in the inlet stream. Above the CRSD, drastic reductions in core permeability occur due to a $\log \mathrm{jam}$, or bridging effect, at the pore constriction. Below the CRSD, the released clay particles pass through the constriction without being captured and therfore very little reduction in core permeability occurs. A critical particle concentration was also identified above which bridging occurs. Based on the experimental observations, a mathematical model was developed to delineate the regions of high and low permeability reductions. The model predictions are in good agreement with experimental measurements.
\end{abstract}

\section{INTRODUCTION}

The permeability of clay sandstones decreases rapidly and significantly when the salt water present in the sandstone is replaced by fresh water. The sensitivity of the sandstone to fresh water is primarily due to clay particle migration, which is the focus of this work [1-5].

Water sensitivity is a factor in the reduction of gas and oil production in many sandstone formations. Although water sensitivity was first observed during water flooding of petroleum reservoirs, it is now a serious concern in many other areas. Field operations such as drilling and solution mining which require the use of aqueous solutions may cause permeability reduction in sensitive sandstones. For example, attempts to acidize wells with aqueous solutions of $\mathrm{HCl}$ and HF can produce significant reductions in permeability near the well bore. In addition to many practical applications, the study of the water sensitivity of sandstones is a problem of general scientific interest in colloid chemistry. Colloidal and interfacial phenomena such as flocculation, peptization and adsorption are all factors in the water sensitivity of sandstone.

A standard experiment which demonstrates the water sensitivity of sandstone is shown in Fig. 1. When the salt concentration of the water flowing through the sandstone core was rapidly decreased from $0.51 \mathrm{M}$ to $0.0 \mathrm{M} \mathrm{NaCl}$ (fresh water), the normalized core permeability of the core $\left(K / K_{0}\right)$ was reduced by a factor of 200 . The permeability reduction occurs after only a few pore volumes of fresh water are sent through the core. If the direction of fresh water flow is reversed, i.e. opposite to the direction of flow of the original salt

†Present address: Dept. of Chemical Enginecring, Indian Institute of Technology, Bombay.

*To whom correspondence should be addressed. water solution, the permeability increases temporarily. When the flow is switched from fresh water to salt water, still flowing in the same reverse direction, no change in permeability is observed. However, if salt water flow is reversed the permeability increases permanently (see Fig. 1). These permeability trends suggest that flocculation and peptization of clay particles are occurring, and the permeability reduction is due to the blocking of pores of the sandstone [6].

\section{PREVIOUS WORK}

The previous literature on this rate effect primarily consists of the work of Jones [5] and Mungan[3]. Jones [5] carried out two types of experiments. In one experiment the salt concentration was decreased in a single step. In the other experiment, the salt concentration was decreased by the same total amount but in a number of small steps. The reduction in permeability was significantly less in the series of stepwise decreases in salinity than that which occurred in the single step decrease experiment. Mungan [3] obtained similar results by decreasing the salt concentration in a slow and continuous manner using a continuously stirred mixing vessel. In Mungan's experiment, the permeability of the core was reduced by only $3 \%$ of the original value. Such a small reduction in permeability was indeed negligible compared to the reduction of more than $99 \%$ when the salt concentration was decreased abruptly. Jones [5], Mungan [3, 4] and Gray[2] explained this finding by assuming that clay particles are not released when the salt concentration is decreased slowly. Jones attributed the cause of particle release to the osmotic pressure developed by the difference in the salt concentration in the space between the clay particles and the bulk solution. When the bulk salt concentration decreases slowly, the difference in the salt concentrations remains low and thus less osmotic pressure is developed to release 


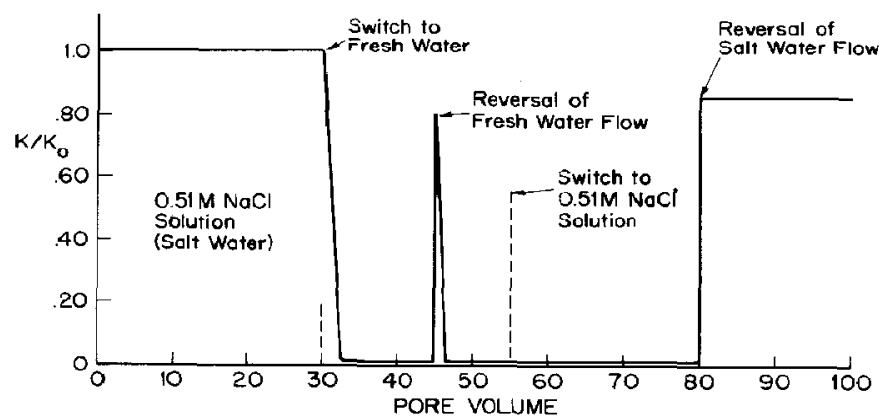

Fig. 1. Permeability reduction and permanent permeability gain in watcr sensitivity of Bcrea sandstone.

the clay particles. However, this osmotic hypothesis was refuted by Mungan [3] who showed experimentally that the osmotic pressure was not the cause of particle release. Before Mungan's work [4], Gray [2] also disagreed with Jones concerning this osmotic pressure hypothesis. Gray proposed that it was the expansion of the double layers of the clay particles that caused these particles to be released. Mungan, meanwhile, proposed that it was the degree of hydration of the sodium ions that caused the permeability reduction in one case and not in the other.

Neither of these last two applications of double layer theory can accurately explain the observation. The double layer force does not depend on the rate at which the sult concentrution is decreased, rather it is strongly dependent on the salt concentration itself. The sensitivity phenomemon appears to be rate dependent with respect to the bulk sodium chloride concentration, e.g. if the rate of the salinity decrease is sufficiently slow, the permeability does not decrease significantly. Regardless of the rate of decrease, both step experiments begin and end at the same concentration. Consequently explanations based solely on double layer theory are inadequate, and need to incorporate the rate at which the salt concentration is decreased.
The assumption that clay particles are not released as the salinity is decreased slowly is unsubstantiated. Nevertheless, the rate dependence of permeability reduction implies that the processes of release and capture of clay particles are dependent on the rate of salinity decrease. This research was carried out to determine whether the processes of clay particle release and capture are dependent on the rate of decrease in salinity and thus whether or not the permeability reduction is rate dependent.

\section{EXPERIMENTAL APPARATUS AND PROCEDURE}

Experiments were conducted using a core-flood type of experimental apparatus in which a continuously stirred mixer was used to slowly decrease the salt concentration.

A schematic diagram of the apparatus is shown in Fig. 2. A twin cylinder Ruska proportionating pump was used to supply sodium chloride solution and fresh water at a constant flowrate. In the fresh water line, a constant stirred mixer was placed in a parallel stream. The rate of salinity decrease depends on the ratio of the volume $(V)$ of the mixer to the flowrate, $(q)$ of the stream. The space velocity, $S=(q / V)$ is a quantitative measure of the rate of salinity change; with large space

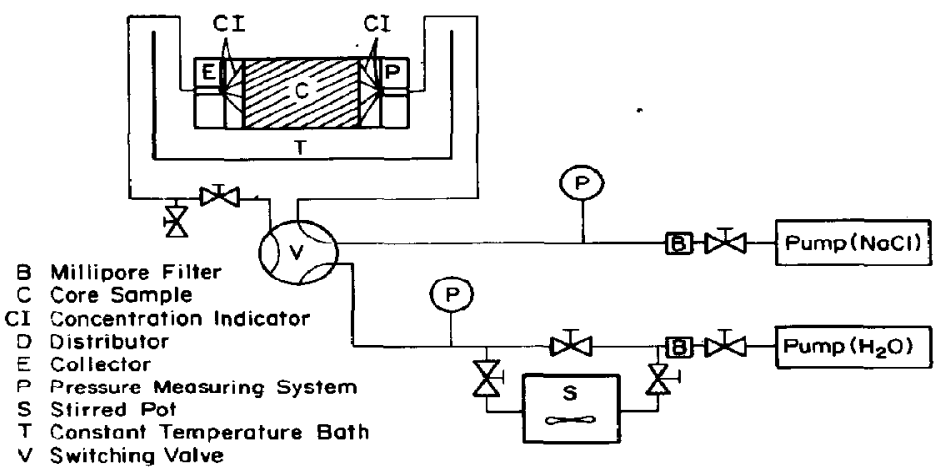

Fig. 2. Schematic diagram of experimental apparatus. 
velocities corresponding to rapid changes in the salt concentration. The reciprocal of the space velocity is the space time, $T$, of the mixer. Mixers of volume ranging from 30 to $2000 \mathrm{~cm}^{3}$ were used, thus the rate of decrease in salt concentration was varied at a given flowrate. The effluent salt concentration, $C$, from the mixer decreased exponentially with time according to the equation $C=C_{0} \mathrm{e}^{-s t} \equiv C_{0} \mathrm{e}^{-\tau / \mathrm{r}}$.

The fluid was forced through two Millipore submicron filters and a plexiglass distributor at the core faces. A plexiglass collector was used to collect the fluid exiting the core. The distributor and the collector also supported four pairs of electrodes which were used in conjunction with a Barnstead water purity meter to monitor the changes in the salt concentration of the fluid.

The sandstone core, the distributor and the collector were assembled in a 2 in. tygon tube which was placed in a core holder. The core holder was immersed in a constant temperature bath. A Unimeasure strain gauge type adaptor and transducer unit were used to measure the pressure drop across the core. A wide range of adaptors were used $(0-8,0-20$, $0-100$ and $0-500 \mathrm{psi})$ to ensure accurate measurement of the pressure drop. An Omniscribe dual channel strip chart recorder was used to record the pressure drop across the core, and the salt concentration both at the inlet and at the outlet.

While Berea sandstone cores were used in all experiments reported in this paper, Boise sandstone exhibited similar water sensitive behavior. Berca sandstone contains about $8 \%$ by weight of dispersable clays such as kaolinite and illite. The porosity of this sandstone is $19 \%$.

A Berea core of 1 in. diameter and 1 in. in length was first vacuum saturated with $0.51 \mathrm{M} \mathrm{NaCl}$ solution. The $0.51 \mathrm{M}$ solution was then forced through the core to determine the initial permeability $\left(K_{0}\right)$ of the core. Once $K_{0}$ was determined, usually after passing about 50 pore volumes of $\mathrm{NaCl}$ solution through the core, the flow was then switched so that fresh water entered the mixer and the effluent of the mixer entered the core. The salt concentration in the effluent of the mixer decreases exponentially with time, with the rate of decrease depending on the space velocity $\left(\mathrm{hr}^{-1}\right)$ of the mixer. The flow was maintained until the salt concentration in the core effluent dropped below $1 \mathrm{ppm}\left(10^{-5} \mathrm{M}\right)$ and the pressure drop across the core became constant. Samples of the effluent from the core were collected and stored for clay particle analysis described in the latter part of this paper. The core was then re-saturated and then subjected to an abrupt decrease in salinity (i.e. a water shock experiment) to test whether the core was indeed water sensitive. In all cases the cores were water sensitive.

The permeability $(K)$ was calculated by using Darcy's law for one dimensional laminar flow of a homogeneous fluid through a porous medium. Darcy's law is given by

$$
v=q / A_{c}=\frac{-K}{\mu}\left(\frac{\Delta P}{L}\right)
$$

where $v=$ superficial velocity $(\mathrm{cm} / \mathrm{sec}), q$ flowrate in $\left(\mathrm{cm}^{3} / \mathrm{sec}\right), A_{c}=$ cross sectional area of the medium perpendicular to the flow $\left(\mathrm{cm}^{2}\right), \mu=$ viscosity of the fluid (cp), $(\Delta P)=$ pressure drop across the core, $L=$ length of the medium (cm), $K=$ permeability of the medium (Darcy).

A pore volume of fluid is equal to $\phi A_{c} L$, where $\phi$ is the porosity.

\section{RESULTS AND DISCISSION}

4(a). Permeability reduction at various rates of salinity decrease

The mixer apparatus described in the previous section was used to carry out a number of experiments in which the salt concentration was decreased in an exponential manner. The flowrate was varied by a factor of approx. 20 while the rate of salinity decrease, characterized by the space velocity of the mixer, was varied by a factor of approx. 100 for a given flow rate. The results are presented in Table 1.

Table 1. Summary of results

\begin{tabular}{|c|c|c|c|c|c|}
\hline $\begin{array}{c}\text { Experimental } \\
\text { Run \# }\end{array}$ & $\begin{array}{c}\text { Flowrate (q) } \\
\mathrm{cc} / \mathrm{sec}\end{array}$ & $\begin{array}{c}\text { Superficial } \\
\text { velocity } \\
\text { cm/hr }\end{array}$ & $\begin{array}{c}\text { Volume (V) } \\
\text { of mixer } \\
\text { ce }\end{array}$ & $\begin{array}{c}\text { space }(-1) \\
\text { velocity } \\
h r^{-1}\end{array}$ & $\left(\mathrm{~K} / \mathrm{K}_{\mathrm{O}}\right)_{\mathrm{E}}$ \\
\hline $\begin{array}{l}\text { R1 } \\
\text { R2 } \\
\text { R3 } \\
\text { R4 }\end{array}$ & $\begin{array}{l}1.67 \times 10^{-3} \\
8.89 \times 10^{-3} \\
8.89 \times 10^{-3} \\
2.22 \times 10^{-2}\end{array}$ & $\begin{array}{r}1.2 \\
6.3 \\
6.3 \\
15.8\end{array}$ & $\begin{array}{r}150 \\
150 \\
150 \\
36\end{array}$ & $\begin{array}{l}0.040 \\
0.222 \\
0.222 \\
2.22\end{array}$ & $\begin{array}{l}0.45 \\
0.67 \\
0.56 \\
0.008\end{array}$ \\
\hline $\begin{array}{l}\text { R5 } \\
\text { R6 } \\
\text { R7 } \\
\text { R8 } \\
\text { R9 } \\
\text { R10 } \\
\text { R11 }\end{array}$ & $\begin{array}{lll}3.61 & \times & 10^{-2} \\
3.61 & \times 10^{-2} \\
3.61 & \times 10^{-2} \\
3.61 & \times & 10^{-2} \\
3.61 & \times 10^{-2} \\
3.61 & \times 10^{-2} \\
3.61 & \times & 10^{-2}\end{array}$ & $\begin{array}{l}25.6 \\
25.6 \\
25.6 \\
25.6 \\
25.6 \\
25.6 \\
25.6\end{array}$ & $\begin{array}{r}1.600 \\
1.080 \\
530 \\
385 \\
255 \\
100 \\
36\end{array}$ & $\begin{array}{l}0.083 \\
0.125 \\
0.263 \\
0.357 \\
0.555 \\
1.320 \\
3.85\end{array}$ & $\begin{array}{l}0.70 \\
0.67 \\
0.62 \\
0.16 \\
0.01 \\
0.008 \\
0.008\end{array}$ \\
\hline $\begin{array}{l}\text { Rl } 2 \\
\text { Rl } 3 \\
\text { Rl } 4 \\
\text { Rl } 5\end{array}$ & $\begin{array}{l}3.33 \times 10^{-3} \\
3.33 \times 10^{-3} \\
3.33 \times 10^{-3} \\
3.33 \times 10^{-3}\end{array}$ & $\begin{array}{l}2.4 \\
2.4 \\
2.4 \\
2.4 \\
\end{array}$ & $\begin{array}{r}148 \\
65 \\
36 \\
24 \\
\end{array}$ & $\begin{array}{l}0.082 \\
0.212 \\
0.345 \\
0.526\end{array}$ & $\begin{array}{l}0.36 \\
0.11 \\
0.02 \\
0.008\end{array}$ \\
\hline
\end{tabular}




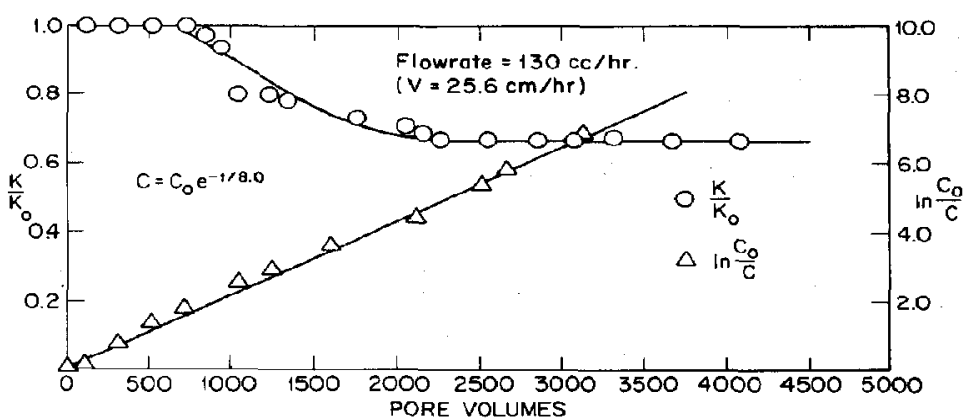

Fig. 3. Permeability reduction and salt concentration at a space velocity of $0.125 \mathrm{hr}^{-1}$.

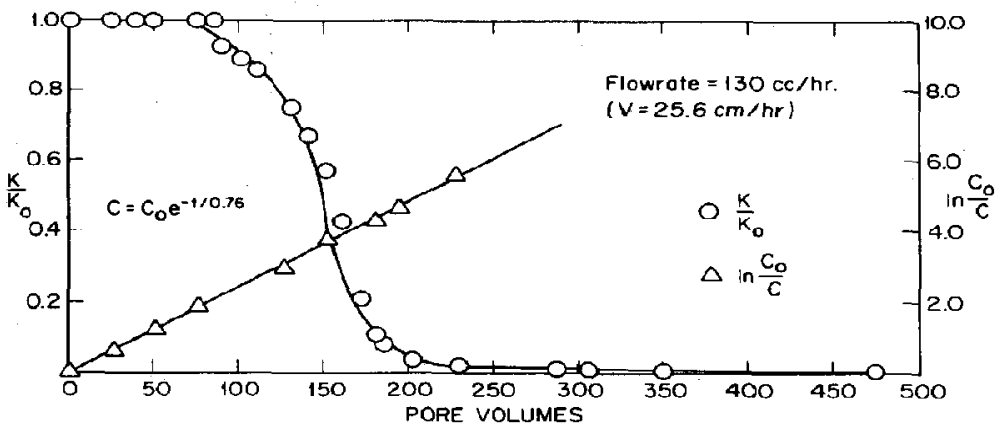

Fig. 4. Permeability reduction of salt concentration at a space velocity of $1.316 \mathrm{hr}^{-1}$.

Typical plots showing the variation of the reduced permeability $\left(K / K_{0}\right)$ and the salt concentration with pore volume throughput of mixer and effluent are shown in Figs. 3 and 4. The reduced permeability $\left(K / K_{0}\right)$, is shown as a function of cumulative pore volumes passed through the core. The effluent salt concentration is shown on a logarithmic scale. The salt concentration plots were straight lines in all cases. The slopes of these straight lines were used to compute the mixer residence time, $T$, which is the inverse of space velocities tabulated in Table 1 . The space velocities are either equal to or slightly greater than the corresponding theoretical values. The theoretical residence times are computed by taking the ratio of the flowrate, $q$, to the volume of the mixer, $V$.

Figure 3 shows the permeability reductions in an experiment in which the salt concentration was decreased rather slowly. As one notices in this figure, the reduction in permeability is only $30 \%$ which is indeed negligible compared to the reduction by a factor of approx. 200 resulting from an abrupt decrease in salinity (see Fig. 1). However, Fig. 4 shows that at a high rate of decrease, the core permeability is reduced drastically. Such a drastic reduction in permeability usually occurs during an abrupt decrease in salinity, for instance, when the flow is switched from salt to fresh water. In fact, it was found that permeability reduction increases with increasing rate of decrease, which can be inferred from the results presented in Table 1 . In addition, these results indicate the existence of a critical rate of salinity decrease (CRSD). When the rate of decrease is above the CRSD, drastic permeability reduction occurs. Below the CRSD, the core permeability remains virtually unreduced.

\section{4(b). Critical rate of salinity decrease (CRSD)}

The CRSD was obtained by plotting the final permeability reduction as a function of space velocity (Fig. 5). These plots were obtained at two superficial velocities 25.6 and $2.4 \mathrm{~cm} / \mathrm{hr}$.

As one observes in Fig. 5 , the CRSD can be taken at any value between 0.30 and $0.40 \mathrm{hr}^{-1}$ at the higher fluid velocity of $25.6 \mathrm{~cm} / \mathrm{hr}$. If the salt concentration decreased at some rate greater than the rate corresponding to the space velocity of $0.40 \mathrm{hr}^{-1}$, the core permeability was drastically reduced. Thus, the rate of decrease in salinity is also an important consideration in preventing permeability reduction caused by clay particle migration. This plot also includes Mungan's results [3], which are in agreement with those in the present work.

At the lower superficial velocity, $2.4 \mathrm{~cm} / \mathrm{hr}$, the permeability reductions, in general, were found to be lower than those at the higher flow rate. For 


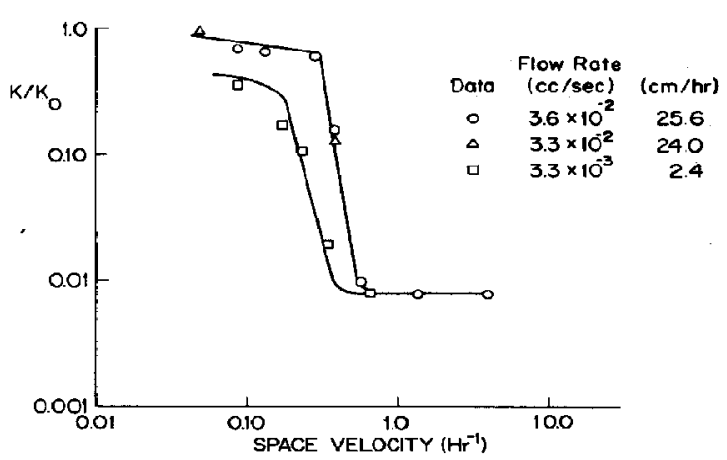

Fig. 5. Critical rate of salinity decrease in water sensitivity of Berea sandstone.

example, at a rate of salinity change corresponding to the space velocity of $0.30 \mathrm{hr}^{-1}$, the permeability ratio $K / K_{0}$ for $2.4 \mathrm{~cm} / \mathrm{hr}$ is about 0.02 which is about 25 times smaller than the $K / K_{0}$ of 0.50 at the higher velocity. As can be seen in Fig. 5 , it is difficult to assign a precise value to the CRSD at the lower superticial velocity. The CRSD can be taken as approx. $0.10 \mathrm{hr}^{-1}$ at a flow rate of $2.4 \mathrm{~cm} / \mathrm{hr}$, a flowrate comparable to flowrates used in gas and oil production.

\section{4(c). Results of effiuent analysis}

The effluent from the sandstone core was analyzed both microscopically and chemically. Scanning electron micrographs were taken of core effluent samples collected, experiments R6 and R11, to determine if clay particles are released when the salt concentration is decreased at a slower rate. The effluent samples of experiments $R 6$ and $R 11$ were chosen because the rate of salinity decrease and the permeability ratio $K / K_{0}$ of these two experiments corresponds to the extremes of the respective ranges (see Fig. 6). Therefore, it is necessary to compare the clay particle concentrations in the effluents of these two experiments. Approximately one cubic centimeter of effluent was filtered through a $0.6 \mu$ Nucleoporc filter paper. The particles on the filter paper were then dried, and used for taking SEM micrographs. Clay particles were observed in both micrographs in Fig. 6. The micrograph of R11 (high rate of salinity change) shows a much larger concentration of clay particles than that shown in the micrograph of R6. While the concentration of clay particles is low, one observes from Fig. 6 that the total amount of clay particles released is significant. One concludes that clay particles are released regardless of the rate of salinity decrease. Therefore, in contradiction to previous works stating that particles are not released at a slow rate of decrease in sulinity, we find particles are indeed released. One can also conclude that particles are released rapidly in experiment $R 11$ $\left(S=3.85 \mathrm{hr}^{-1}\right)$. To quantify this, the clay particle concentrations were measured.

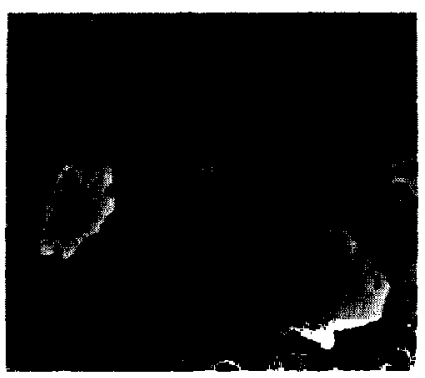

(a)

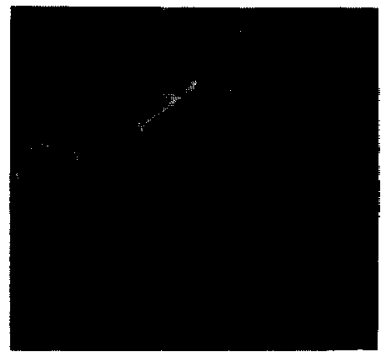

(b)

Fig. 6. Electron micrographs showing clay particles in the eftiuents. (a) Clay particles in effluent of experiment R6 $\left(0.125 \mathrm{hr}^{-1}\right)$. (b) Clay particles in effluent of experiment $R 11$ $\left(3.85 \mathrm{hr}^{-1}\right)$.

The analytical technique used to measure clay particle concentration consisted of dissolving clay particles in HF $(6.0 \mathrm{~N})$ to yield mostly silicon and aluminum ions $\left(\mathrm{Si}^{4+}, \mathrm{Al}^{3+}\right)$. The samples of dissolved clay particles were analyzed for these ions and other cations such as a $\mathrm{Ca}^{2}{ }^{1}, \mathrm{Mg}^{2+}$ and $\mathrm{K}^{+}$with an atomic absorption spectrometer. The concentrations of $\mathrm{Ca}^{2+}, \mathrm{Mg}^{2+}$ and $\mathrm{K}+$ ions ranged from 1 to $4 \mathrm{ppm}$, 0 to $1 \mathrm{ppm}$ and 1 to $4 \mathrm{ppm}$ respectively. The presence of such low concentrations of these ions is not likely to counteract the effect of decreasing the $\mathrm{Na}^{+}$ion concentration. It was assumed that most of the migrating clay particles are kaolinite $\left((\mathrm{OH})_{8} \mathrm{Si}_{4} \mathrm{Al}_{4} \mathrm{O}_{10}\right)$ which accounts for virtually all the clay content of Berea sandstone. Based on this assumption the $\mathrm{Al}^{3+}$ concentrations were converted to clay particle concentrations.

Figures 7-9 show the clay concentration in the effluent stream as a function of pore volumes of fluid passed through the core at different space velocities and flow rates. These figures also contain information on the salt concentration and the permeability reduction. The important features of these figures are:

(1) No clay particles are observed in the effluent solution from the core until the salt concentrution falls below the critical salt concentration (CSC) (Fig. 6). After the CSC is reached the concentration of clay particles increases, goes through a maximum and then decreases. 


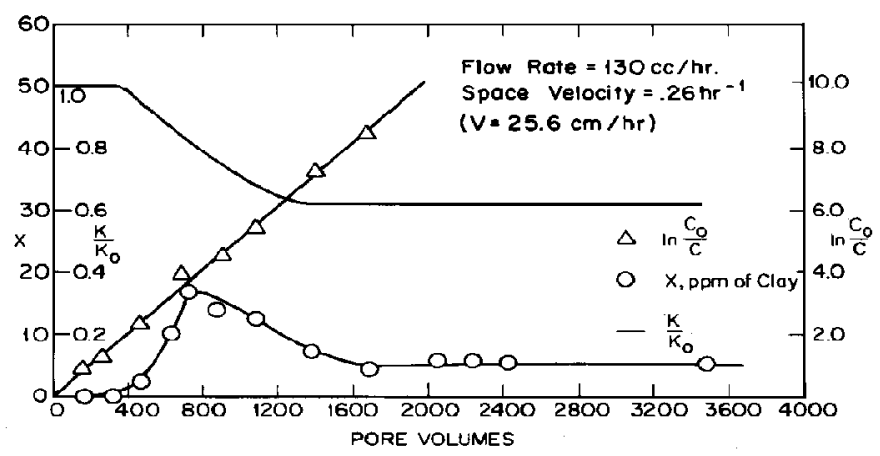

Fig. 7. Clay concentrations at slow decrease in salt concentration.

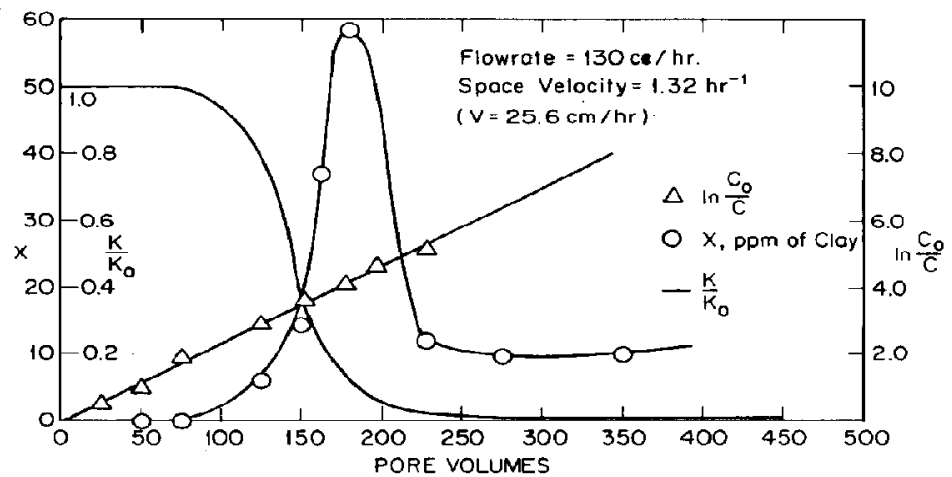

Fig. 8. Clay concentration at fast decrease in salinity.

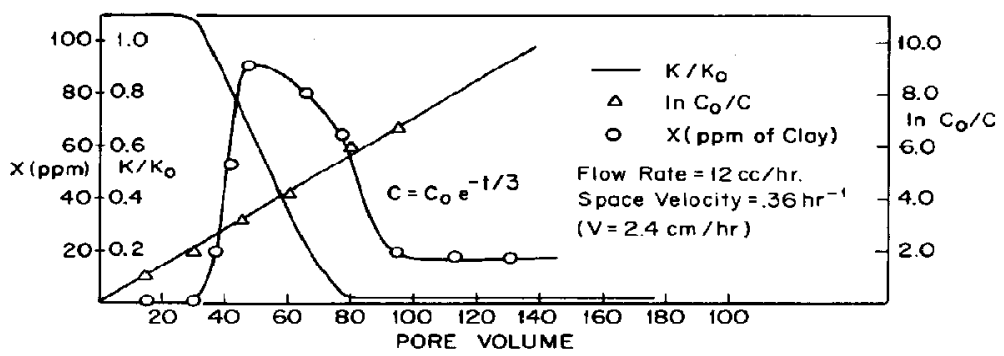

Fig. 9. Clay concentrations at lower flow rate.

(2) The greater the space velocity is the greater the clay concentration in the effluent will be (Fig. 7 vs Fig. 8). At a space velocity of $0.26 \mathrm{hr}^{-1}$ the concentration of clay particles is about 4-5 times that for the space velocity of $1.33 \mathrm{hr}^{-1}$.

(3) The lower the superficial velocity the greater is the clay concentration in the effluent for the same rate of salinity decrease. The effluent clay concentration for a flowrate of $2.4 \mathrm{~cm} / \mathrm{hr}$ was 5-6 times higher than the concentration at a flowrate of $25.6 \mathrm{~cm} / \mathrm{hr}$.
The first observation of a critical salt concentration occurs as a result of electric double layer interactions and is explained elsewhere [6]. The last two observations can be explained in the following manner. The concentration of clay particles in the core effluent depends on the amount of particles released in a given time as well as the amount of fluid forced through the core during this period of time. Approximately the same amount of clay particles are released irrespective of the flowrate and the rate of salinity 
Table 2. Mass of clay particles at various space velocities and superficial velocities

\begin{tabular}{|c|c|c|}
\hline $\begin{array}{c}\text { Space Velocity } \\
\text { (hr) }\end{array}$ & $\begin{array}{c}\text { Superficial Velocity } \\
(\mathrm{cm} / \mathrm{h} x)\end{array}$ & $\begin{array}{l}(\Delta M) \\
\text { (gms) }\end{array}$ \\
\hline 0.125 & 25.6 & 0.025 \\
\hline 0.263 & 25.6 & 0.022 \\
\hline 9.34 & 2.4 & 0.020 \\
\hline
\end{tabular}

change. This experimental fact is shown in Table 2 .

The quantity $(\Delta M)$ is the amount collected until the salt concentration is reduced to $75 \mathrm{ppm}$.

Consequently, the clay particle concentration in the core effluent will be inversely proportional to the amount of fluid sent through the core. For example, if more fluid is passed through the core, as in the experiment at a higher flowrate, then the concentrations should be low and this is what was observed. Likewise, for a slower rate of salinity change experiment, more fluid must pass through the core to achieve the same decrease in salt concentration, and therefore the concentration of clay particles in the slower salinity change experiment will be lower than that compared to the rapid change experiment.

A comparison of permeability reduction plots with clay concentration plots (Figs. 7-9) shows that the permeability does not begin to decrease until clay particles are observed in the effluent. That is, the clay particles have not begun to release from the pore walls and consequently there is no reduction in core permeability. In Figs. 7 and 8 one observes that the permeability begins to reduce after about 400 and 75 pore volumes, respectively, have passed through the core. This number of pore volumes in each core is required to reduce the salt concentration to approx. $400 \mathrm{ppm}$, the critical salt concentration. One also observes from Figs. 7 and 8 that the decline in core permeability is particularly rapid as the clay concentration increases. This occurs roughly between $\mathbf{4 0 0}$ and 1000 pore volumes in Fig. 7 and between 100 and 175 pore volumes in Fig. 8 corresponding to salt concentrations of $400-500 \mathrm{ppm}$. Furthermore, it can be seen that the decrease of reduction in permeability is strongly dependent on the concentration of clay particles. Thus, the degree of plugging in the porous media depends on the number of clay particles present in the suspension in the pores. Mucke [8] has also shown that the plugging at constrictions depends strongly on the particle concentration.

The mass of the clay particles released can be found by computing the area under the clay concentration curves. Figure 10 shows the mass of clay particles released as a function of the salt concentration. It can be seen in Fig. 10 that the mass of the clay particles released at space velocities of 0.263 and $0.125 \mathrm{hr}^{-1}$ are virtually equal at salt concentrations of 500,75 and $10 \mathrm{ppm}$. However, the mass of clay particles at a space velocity of $1.32 \mathrm{hr}^{-1}$ and a velocity of $25.6 \mathrm{~cm} / \mathrm{hr}$ are consistently lower, since some particles were captured at these conditions. The final

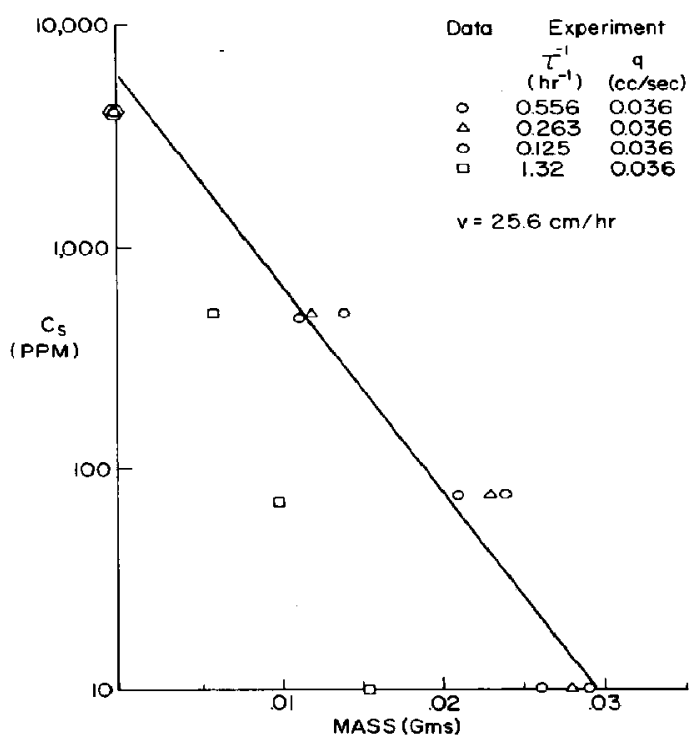

Fig. 10. Amount of clay particles collected at various salt concentrations.

permeability in this core was $2 \%$ of the original value. Consequently, as a first approximation one can conclude that the amount of particles released for a given drop in salt concentration is the same regardless of the flow rate and the rate of decrease in salinity.

The analyses of core effluents show two important results. First, the amount of the particles released depends on the salt concentration. Or, in other words, for a given drop in the salt concentration below the $\mathrm{CSC}$, the same number of clay particles are released regardless of the flowrate. Secondly, the amount of particles captured depends on the concentration of the clay particles in the sandstone which, in turn, depends on the rate at which the salt concentration is decreased.

Based on these experimental observations, a mechanism is proposed to describe the dependence of water sensitivity in sandstone on the rate of salinity change. In the case of an abrupt decrease in the salt concentration a large number of clay particles are releused in a short lime and conseyuently the bulk particle concentration increases rapidly (Fig. 8). As the particle concentration increases, more particles are captured due to bridging or "log-jams" at the pore throat and thus permeability is reduced 


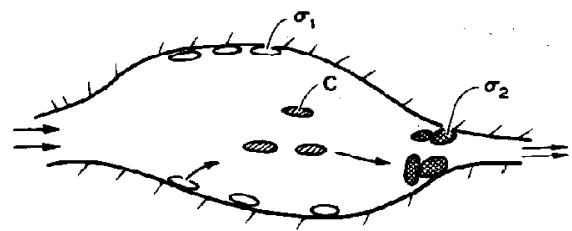

$\bigcirc$ Porticles ottached to pore wall P. Porticles in suspension $\$$ Captured particles

Fig. 11. Schematic diagram showing particle bridging.

significantly (Fig. 11). Conversely as the salt concentration is decreased slowly, the clay particles are released over a longer period of time. The particle concentration remains lower and many clay particles do not arrive at the pore throat at the same time to cause a "log-jam" effect. Therefore, the number of clay particles captured is lower and the reduction in permeability is also lower. Occurrences similar to the log-jam have been observed in ultrafiltration[9] as well as in traffic flow on a route consisting of "bottlenecks" [10]. More importantly, it has been shown that there exists a jam concentration or critical concentration above which the bridging occurs.

\section{A MODEL FOR CRSD}

A model was developed to predict the critical rate of salinity decrease (CRSD) and the predictions are in good agreement with the experimental data. The basis of the model is the mechanism described in the first section of this paper.

The CRSD is a useful parameter in the study of water sensitive sandstones. If the rate of salinity decrease is below the CRSD, then there is very little or no reduction in core permeability. If the rate of decrease is higher than the CRSD, then the core permeability will be drastically reduced. Therefore, the dependence on the rate of salinity decrease for all practical purposes can be completely described if the critical rate of salinity decrease is known.

In our model for watershock, a sandstone core is considered to consist of a number of connecting segments. In addition to the release and capture of the clay particles in a segment [6], there is convective transport of clay particles from one segment to another. However, this convective transport of particles takes place only at low concentrations of the particles. At high particle concentrations, there is virtually no transport of clay particles between the segments [6]. Therefore a critical concentration $\left(C_{P C}\right)$ exists above which the particle concentrations are high enough to create bridging of particles across the pore throat. As a result, there is very little transport of clay particles between the segments.

5(a). Calculations of the effuent particle concentration A schematic diagram for the $i$ th segment is shown in Fig. 12. A differential material balance on the clay

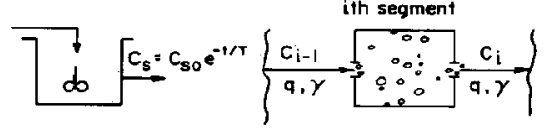

Fig. 12. Schematic diagram of the system in exponential decrease experiment.

particles in the $i$ th segment yields

$$
\gamma\left(q C_{i-1}-q C_{i}\right)+r_{n} V-r_{c i} V=V \frac{\mathrm{d} C_{i}}{\mathrm{~d} t}
$$

where $C_{i}=$ concentration of particles in the $i$ th segment, $r_{r i}=$ rate of release in the $i$ th segment, $r_{c i}=$ rate of capture in the $i$ th segment, $\gamma=$ fraction of particles leaving the segment with the flow, assumed to be the same for each segment

$$
\left(\gamma_{i}=\gamma_{i-1}\right)
$$

Dividing throughout by $q$, one has

$$
\gamma\left(C_{i-1}-C_{i}\right)+\tau r_{r i}-\tau r_{c i}=\tau \frac{\mathrm{d} C_{i}}{\mathrm{~d} t} .
$$

The capture rate equation has been developed previously using the concept of the deposition of a particle on a spherical collector[6]. Thus it has been of the form:

$$
\frac{\mathrm{d} \sigma_{2 i}}{\mathrm{~d} t}=\beta C
$$

where $\sigma_{2 i}$ is the concentration of particles captured in the $i$ th segment and $\beta$ is the capture coefficient.

The rate of release of particles is assumed to be of the form [6]

$$
r_{r i}=-\frac{\mathrm{d} \sigma_{1 i}}{\mathrm{~d} t}=\alpha_{i} \sigma_{1 i}
$$

where $\alpha_{i}$ is the release coefficient in the $i$ th segment and $\sigma_{1 i}$ is the instantaneous concentration of clay particles present on the pore wall of the $i$ th segment. Equation (3) now becomes:

$$
\gamma\left(C_{i-1}-C_{i}\right)+\tau \alpha_{i} \sigma_{1 i}-\tau \beta C_{i}=\tau \frac{\mathrm{d} C_{i}}{\mathrm{~d} t}
$$

It is known that the amount of clay particles released is dependent on the salt concentration (Fig. 12). Therefore, the release coefficient $\alpha$ which partly governs the number of particles to be released should depend on the salt concentration. In the water shock model and experiment, a time averaged release coefficient $\alpha$ was assumed constant. However, one cannot make this assumption when the release of clay particles takes place over a long period of time. Nevertheless, a linear relationship relating the release coefficient and the salt concentration was assumed. 


$$
\begin{aligned}
& \alpha=0 \\
& \text { for } C_{s}>\mathrm{CSC} \\
& \alpha=\alpha_{0}\left(1-\frac{C_{s}}{\mathrm{CSC}}\right) \text { for } C_{s}<\mathrm{CSC}
\end{aligned}
$$

$\alpha_{0}$ is the maximum value of the release coefficient realized at very low salt concentrations. The release coefficient $\alpha$ increases as the salt concentration $\left(C_{s}\right)$ decreases. Before the salt concentration, $C_{s}$, reaches the CSC, no particles are released and $\alpha$ is zero [6]. As the $C_{s}$ decreases below the $\mathrm{CSC}$, larger double layer repulsive interactions occur and therefore, the release of particles is rapid.

The salt concentration is a function of time. At the inlet, it is given by

$$
C_{s i}=C_{s o} \mathrm{e}^{-t_{i} / T}
$$

where $C_{50}$ is the initial salt concentration in the mixer and $T$ is the residence time of the mixer. Since the residence time of the mixer $(T)$ in all experiments conducted in this work was between 100 and 5000 times greater than the residence time of a segment $\tau$, the salt concentrations in all segments of a core of 1 in. length can be assumed to be equal to that in the effluent $\left(C_{s}\right)$ of the core. The salt concentration in the core effluent was found to vary exponentially as shown below

$$
C_{s}=C_{s 0} \mathrm{e}^{-t / T}
$$

where $1 / T$ is the slope of the salt concentration plot in Figs. 7-9. If the time $(t)$ is measured from the instant $C_{s}$ reaches the $\mathrm{CSC}$, one then has

$$
C_{s} / \mathrm{CSC}=\mathrm{e}^{-t / T}
$$

substituting for $C_{s} / \mathrm{CSC}$ in the equation (6b)

$$
\begin{array}{ll}
\alpha_{i}=0 & \text { for } C_{s}>\mathrm{CSC} \\
\alpha_{i}=\alpha_{0}\left(1-\mathrm{e}^{-t_{i} / T}\right) & \text { for } C_{s}<\mathrm{CSC} \\
i=1,2, \ldots n &
\end{array}
$$

where $n$ is the number of segments, $t_{i}$ is the time measured from the instant the CSC is reached in the $i$ th segment. Equation (5) can now be readily integrated with the condition $\sigma_{1 i}=\sigma_{10}$ at $t=0$ to yield

$$
\sigma_{1 i}=\sigma_{10} \exp \left[-\alpha_{0} T\left(-1+t_{i} / T+\mathrm{e}^{-t_{i} / T}\right)\right] .
$$

The experimental measurements of the concentration of clay particles and the permeability reduction suggest that a critical particle concentration $\left(C_{p c}\right)$ exists below which very few particles are captured. Consequently, virtually all the particles are convectively transported out of a segment if the clay concentration is below the critical particle concentration, $C_{p c}$. The $C_{p c}$ is analogous to the jam concentrations in the traffic flow on a route consisting of bottlenecks[10]. Based on the concept of the critical particle concentration, one has

$$
\begin{aligned}
& \beta=0 \\
& \gamma=1 \\
& \beta=\beta \\
& \gamma=0
\end{aligned} \quad \text { for } \quad C_{i}<C_{p c}
$$

The quantitative description of this variation is not known and realistically, the fraction of particles flowing out of the cell $\gamma$ should vary with the amount of captured particles. However, an empirical form for the variation of $\gamma$, similar to that used in filtration may unnecessarily introduce more empirical parameters. Nevertheless, a unit step type of functionality was used to circumvent this problem and was found to yield satisfactory agreement with experimental observations.

Substituting the release and capture rates from the eqns (9), (10) and (4) in eqn (4), one has

$$
\begin{gathered}
\tau \frac{\mathrm{d} C_{i}}{\mathrm{~d} t}+(\gamma+\beta \tau) C_{i}=\gamma C_{\mathrm{i}-1}+\tau \alpha_{0}\left(1-\mathrm{e}^{-t / T}\right) \\
\times \sigma_{\mathrm{t} 0} \exp \left[-\alpha T\left(-1+t / T+\mathrm{e}^{-t / T}\right)\right] \\
C_{i}=0 \text { for } t=0 \text { and } i=0 .
\end{gathered}
$$

Dedimensionalizing,

$$
\theta=t / T, \quad \psi_{i}=C_{i} / \sigma_{10}
$$

one has,

$$
\begin{aligned}
\frac{\mathrm{d} \psi_{i}}{\mathrm{~d} \theta} & +\left(\frac{T}{\tau}\right)(\gamma+\beta \tau) \psi_{i}=\left(\frac{\mathrm{T}}{\tau}\right) \gamma \psi_{i-1}+\alpha_{0} T\left(1-\mathrm{e}^{-\theta}\right) \\
& \times \exp \left[-\alpha_{0} T\left(-1+\theta+\mathrm{e}^{-\theta}\right)\right]
\end{aligned}
$$

with the initial and entrance conditions

$$
\begin{aligned}
& \begin{array}{r}
\psi_{i}=0 \\
\psi_{0}=0
\end{array} \text { for } \quad t=0 \\
& \text { for } \begin{aligned}
\psi_{i}<\psi_{p c} & \quad \beta=0 \\
\gamma & =1
\end{aligned} \\
& \text { for } \begin{array}{rlrl}
\psi_{i}>\psi_{p c} & \quad \beta & =0 \\
\gamma & =1
\end{array}
\end{aligned}
$$

Equations (11) can be used to compute the dimensionless particle concentration $\left(\psi_{i}\right)$ as a function of $\theta$ at various rates of salinity decrease $(S=(1 / T))$. Consequently, knowing $\psi(\tau)$ and $\sigma_{10}$ one can then compute the clay particle concentration as a function of cumulative pore volumes at various rates of salinity decrease. The parameters of the model required for the computation were obtained in the following manner. The values of the capture coefficient obtained for water shock experiments were used in this analysis. For example, $\beta$ is equal to $1.5 \times 10^{-2} \mathrm{sec}^{-1}$ at a superficial velocity of $25.6 \mathrm{~cm} / \mathrm{hr}$. However, the values of $\alpha_{0}$ are not equal to the values of $\alpha$ obtained in the watershock calculations, since $\alpha$ represents a 
time-average value of the salt concentration dependent release coefficient. An estimate of the release coefficient $\alpha_{0}$ can be found from the equation given below [6]

$$
\alpha_{0} T \int_{2}^{10}\left(1-e^{-\theta}\right) d \theta=\alpha_{\text {shock }} \times(\text { time of shock). }
$$

The above equation was derived by equating the total amount of particles released in a typical slow decrease experiment to that in a watershock experiment. The total amount of particles released was obtained by integrating eqn (5). The limits of integration 2-10 represent respectively the initiation of the particle release and the termination of the slow decrease experiment.

Taking typical values of $T=10^{4} \mathrm{sec}$ for the slow decrease experiment, and time of shock equal to two pore volumes at the flowrate of $25.6 \mathrm{~cm} / \mathrm{hr}, \alpha_{0}$ is approximately equal to $4.0 \times 10^{-5} \mathrm{sec}^{-1}$. The dimensionless critical particle concentration is:

$$
\psi_{p c}=\frac{C_{p c}}{\sigma_{10}}
$$

The critical particle concentration, $C_{p e}$, can be taken as the average clay particle concentration in the effluent of the experiment conducted at a rate just below the CRSD. The $C_{p c}$ was taken as the average concentration of the clay particles in the experiment conducted at a rate below the CRSD and is equal to $10 \mathrm{ppm}\left(10^{-5} \mathrm{gm} / \mathrm{cm}^{3}\right)$. This volume average concentration is a ratio of two quantities.

(1) The area under the clay concentration curve between the point it begins to increase and the point it begins to level off (about 400 and 1600 core volumes in Fig. 7).

(2) The cumulative amount of fluid sent through the core during this time interval.

The concentration of particles can be estimated from Fig. 10 and compared with the previously calculated values [6]. As one sees in Fig. 10 approx. $0.03 \mathrm{gm}$ of clay particles are released over the time it takes the salt concentration to reach a very low value (about $10 \mathrm{ppm}$ ). Therefore, one can assume that the amount of releasable clay particles for a 1 in. diameter and 1 in. long core is $0.03 \mathrm{gm}$. Dividing this amount of $0.03 \mathrm{gm}$ by the void volume of the core $\left(2.5 \mathrm{~cm}^{3}\right)$, the estimate of $\sigma_{10}$ is of the order $10^{-2} \mathrm{gm} / \mathrm{cm}^{3}$. The results concerning clay particle migration in thin cores during the water shock also agree with this estimate of $\sigma_{10}[6]$. Therefore, $\psi_{p c}$ is equal to $10^{-3}$.

It should be pointed out that both $\psi_{p c}$ and $\alpha_{0}$ are essentially independent of the flowrate while $\tau$ and $\beta$ are dependent on the flowrate. The capture coefficient $\beta$ is proportional to the flowrate[6] whereas $\tau$ is inversely proportional to the flowrate.

A typical clay concentration plot as a function of cumulative pore volume is shown in Fig. 13. As one observes in these figures, there is reasonable agreement between the model and the data. The lack of better agreement can be attributed to the uncertainties involved in computing $C_{p c}$ and $\sigma_{10}$.

Theoretical plots of $\psi_{e}$ (the dimensionless concentration in the last segment) at various levels of $S$ are shown in Figs. 14 and 15 at two different flowrates. The model parameters used in these computations are presented in Table 3.

The nature of the curves for the ratio $\psi$ in other segments is the same as that for the last segment, with the exception of the first segment. Three observations are noted from Figs. 14 and 15.

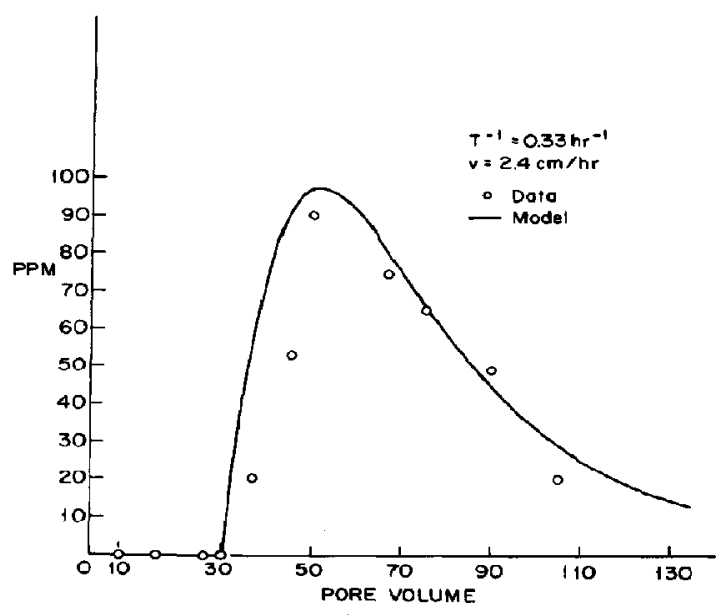

Fig. 13. Comparison of prediction of clay particle concentration with data.

Table 3. Parameters at two flowrates

\begin{tabular}{lr}
$\begin{array}{l}\text { Flowrate }=3.6 \times 10^{-2} \\
c c / \mathrm{sec}\end{array}$ & $\begin{array}{r}\text { Flowrate }=3.34 \times 10^{-3} \\
\text { cc } / \mathrm{sec}\end{array}$ \\
$\alpha_{0}=4 \times 10^{-5} \mathrm{sec}^{-1}$ & $\alpha_{0}=4 \times 10^{-5} \mathrm{sec}^{-1}$ \\
$B=1.5 \times 10^{-2} \mathrm{sec}^{-1}$ & $B=1.5 \times 10^{-3} \mathrm{sec}^{-1}$ \\
$\Psi_{\mathrm{pc}}=10^{-3}$ & $\psi_{\mathrm{pc}}=10^{-3}$ \\
$\tau=14 \mathrm{secs}$ & $\tau=152 \mathrm{secs}$ \\
$\mathrm{n}=5$ & $\mathrm{n}=5$ \\
\hline
\end{tabular}




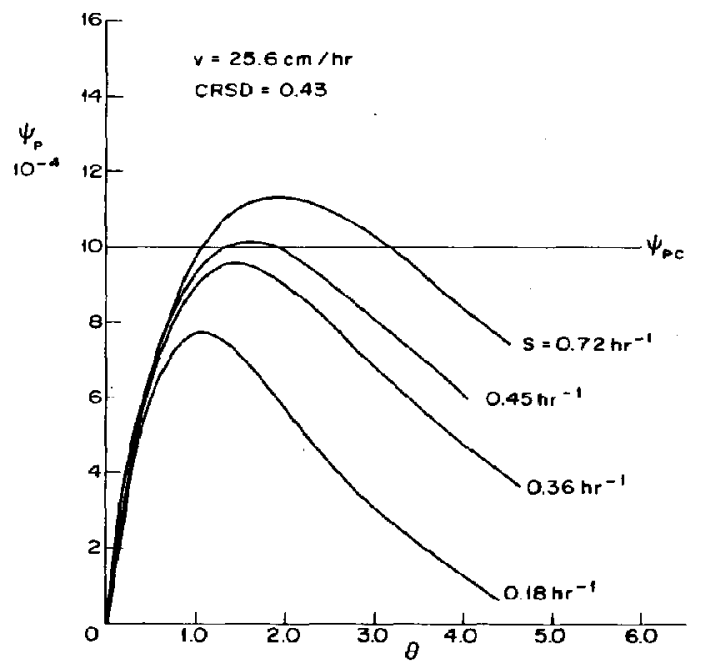

Fig. 14. Dimensionless particle concentration at various space velocities.

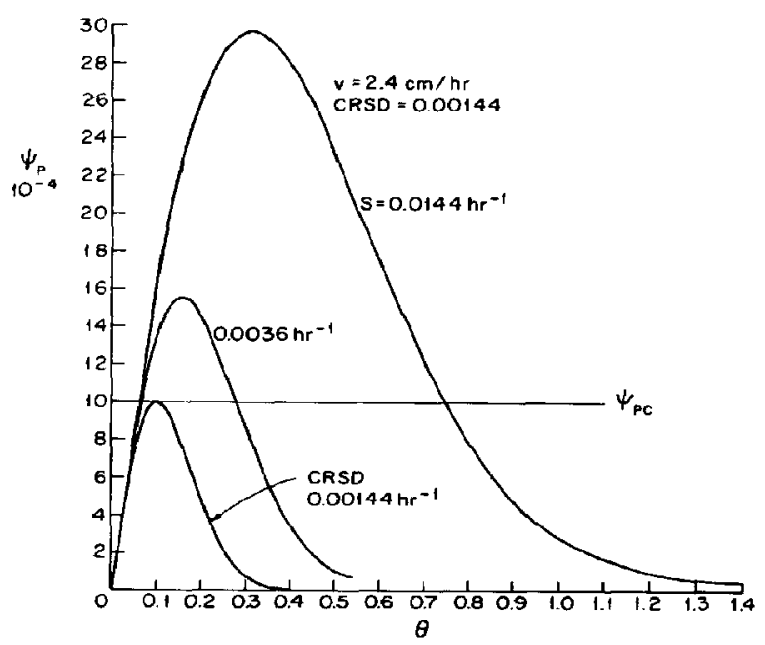

Fig. 15. Dimensionless particle concentration at various space velocities.

(1) The particle concentration increases rapidly from $\theta=0$, which is the time $C_{s}$ becomes equal to the CSC and then goes through a maximum before it begins to decrease.

(2) Higher particle concentrations were obtained for greater rates of salinity decrease. For example, the particle concentrations obtained for a space velocity of $0.124 \mathrm{hr}^{-1}$ were $5-10$ times higher than those obtained for $S=1.29 \mathrm{hr}^{-1}$.

(3) The particle concentrations at the lower flowrates are greater than those at the higer flowrates. The particle concentration at $2.4 \mathrm{~cm} / \mathrm{hr}$ was about 5-10 times higher than that at $25.6 \mathrm{~cm} / \mathrm{hr}$.
These qualitative observations are in excellent agreement with the experiments described earlicr in this paper.

\section{5(b). Estimation of the CRSD}

The critical rate of salinity decrease, $S_{c r}$, can be estimated from $\psi_{e}$ curves presented in Figs. 14 and 15 . The CRSD is the largest space velocity at which the peak of $\psi_{e}$ curve remains less than or equal to the $\psi_{p c}$ (the dimensionless critical particle concentration). In other words, at this rate of decrease in salinity, the particle concentration would essentially remain less than $C_{p c}$ and therefore, the core permeability would not be reduced due to the absence of particle bridging. The particle concentration plot for the last segment was chosen to estimate $S_{c r}$. This choice is due to the fact that the last segment will have the largest concentration compared to those in other segments due to the convective transport of particles.

Scrutinizing Fig. 14, one notices that the CRSD at a superficial velocity of $25.6 \mathrm{~cm} / \mathrm{hr}$ is equal to $0.43 \mathrm{hr}^{-1}$ (see Fig. 5) which is extremely close to the experimental values of $0.3-0.4 \mathrm{hr}^{-1}$. From Fig. 15, the CRSD can be obtained likewise and is equal to $0.014 \mathrm{hr}^{-1}$. This value at a superficial velocity of $2.4 \mathrm{~cm} / \mathrm{hr}$ is smaller than the experimentally obtained value of $0.10 \mathrm{hr}^{-1}$. However, one notices in Fig. 5 that at the lower velocity of $2.4 \mathrm{~cm} / \mathrm{hr}$ the permeability reductions are larger. Even at a space velocity $0.08 \mathrm{hr}^{-1}$ the reduction, about $65 \%$, indicates that the space velocity has to be smaller than $0.08 \mathrm{hr}^{-1}$ to avoid this comparatively higher reduction in permeability. Based on this experimental observation and considering the simplicity of the analysis, the model estimates the CRSD reasonably well. More importantly, the model is consistent with all the experimental observations.

\section{SUMMARY}

It was found that the permeability reduction of Berea sandstone depends strongly on the rate at which the salt concentration is decreased. In fact, a critical rate of salinity decrease (CRSD) exists below which very little reduction to core permeability occurs. If the rate of decrease is above the CRSD, then a drastic reduction in core permeability occurs. The CRSD was found equal to a space velocity of $0.40 \mathrm{hr}$ ' at a superficial velocity of $25.6 \mathrm{~cm} / \mathrm{hr}$. The superficial velocity was found to have a significant effect on the CRSD. The CRSD was found to decrease with decreasing velocity. At a velocity of $2.4 \mathrm{~cm} / \mathrm{hr}$, the CRSD was found to be equal to a space velocity of $0.10 \mathrm{hr}^{-1}$.

The amount of clay particles in the core effluent was measured by first dissolving the effluent in hydrofluoric acid and then determining the concentration of $\mathrm{Al}^{3+}$ and $\mathrm{Si}^{4+}$ ions with an Atomic $\mathrm{Ab}-$ sorption Spectrometer. It was found that the number of clay particles released depends strongly on the salt concentration. In addition, it was concluded that the 
amount of particles plugging the pore constriction depends strongly on the particle concentration of the suspension.

A mechanism was proposed to explain the rate dependence. In this mechanism, it was shown that during an abrupt decrease in salinity, particles are released rapidly. Large numbers of clay particles in the suspension create a $\log$-jam at the pore throat. However, when the salt concentrations are decreased slowly, the particles are released slowly over a long period of time and therefore not many particles jam at the pore throat. A model was also developed based on this mechanism to predict the critical rate of salinity decrease. The predictions are in reasonable agreement with this experimental data.

Acknowledgements-The authors appreciate the support provided by the National Science Foundation Grants NSFG-ENG79-06647 and NSF CPE 8108197 and the support provided by Professor T. S. Brun who fostered one of the author's (HSF) interest in this subject while on sabbatical at the University of Bergen, Bergen, Norway.

\section{REFERENCES}

[1] Johnson N. and Beeson C. M., Trans. AIME 1945160 43.

[2] Gray D. H. and Rex R. W., 14th Nat. Conf. on Clays and Clay Minerals 1966355.

[3] Mungan N., J. Petrol. Tech. 1965171449.

[4] Mungan N., J. Can. Petro. Tech. 19687113

[5] Jones F. O., Jr., J. Petro. Tech. 196416441.

[G] Khilar K. C., Ph.D. Thesis, University of Michigan 1981.

[7] Khilar K. C. and Fogler H. S., Proceedings on Enhanced Oil Recovery. Plenum Press, New York 1981.

[8] Muecke T. W., J. Petrol. Tech. 1979114.

[9] Zahka J. and Mir L., Chem. Engng Prog. December $197753-55$.

[10] Edie C. Leslie, Traffic Science (Edited by D. C. Gazie). Wiley Interscience, New York 1974. 